\title{
Construção de um objeto de aprendizagem a partir da teoria da aprendizagem significativa moderna, usando linguagem de modelagem unificada
}

\author{
Construction of a learning object from the modern significant learning theory, \\ using language unified modeling
}

\section{Construcción de un objeto de aprendizaje a partir de la teoría del aprendizaje significativo moderno, utilizando un lenguaje de modelado unificado}

\author{
Angelo Trein Lucca (angelotlucca@ san.uri.br) \\ Universidade Regional Integrada do Alto Uruguai e das Missões-Uri \\ Antonio Vanderlei dos Santos (vandao@san.uri.br) \\ Universidade Regional Integrada do Alto Uruguai e das Missões-Uri \\ Marcelo Paulo Stracke (stracke@ san.uri.br) \\ Universidade Regional Integrada do Alto Uruguai e das Missões-Uri \\ Rozelaine de Fatima Franzin (rozelaine@san.uri.br) \\ Universidade Regional Integrada do Alto Uruguai e das Missões-Uri
}

\begin{abstract}
Resumo: O uso de teoria de ensino/aprendizagem na elaboração de objetos de aprendizagem de física básica dos cursos de engenharia, muitas vezes não está explícita. Nesse contexto, surge a justificativa de desenvolvimento de objetos de aprendizagem, onde essa teoria seja a base no seu desenvolvimento com significativas contribuições para o Mestrado Profissional de Ensino Científico e Tecnológico. Este artigo tem como objetivo relatar o desenvolvimento de um protótipo de software de ensino, a ser utilizado no componente curricular de circuitos elétricos. A metodologia, quanto à sua natureza, pode ser classificada como aplicada, que se caracteriza pelo seu interesse prático, em que os resultados sejam aplicados ou utilizados imediatamente na solução de problemas ocorridos na realidade. Quanto aos objetivos, pode ser classificada como exploratória. Quanto à abordagem do problema, foi classificada como uma combinação de aspectos da pesquisa qualitativa e quantitativa, em cada etapa do processo de pesquisa. Do ponto de vista dos métodos, a pesquisa foi feita por meio de modelagem e simulação. Este estudo traz contribuições de construir objetos de aprendizagem que possam colaborar com a aprendizagem significativa de conceitos, tanto de física como de matemática, o que se enquadra no propósito de um ensino científico e tecnológico.
\end{abstract}

Palavras-chave: Aprendizagem Significativa; Circuitos Elétricos; Mapas Conceituais; Software.

Abstract: The use of teaching / learning theory in the elaboration of basic physics learning objects in engineering courses is often not explicit. In this context, there is a justification for

Recebido em: 30/05/2021

Aceite em: 13/08/2021 
the development of learning objects, where this theory is the basis for its development with significant contributions to the Professional Masters in Scientific and Technological Education. Therefore, this article aims to report the development of a prototype of teaching software to be used in the curricular component of electrical circuits. The methodology as to its nature, can be classified as applied, which is characterized by its practical interest, in which the results are applied or used immediately in the solution of problems that occurred in reality. As for the objectives, it can be classified as exploratory. As for the approach to the problem, it was classified as a combination of aspects of qualitative and quantitative research at each stage of the research process. From the point of view of the methods, the research was done through modeling and simulation. This study brings contributions to build learning objects that can collaborate with the meaningful learning of concepts, both in physics and mathematics, which fits the purpose of scientific and technological teaching.

Keywords: Learning; Electric circuits; Concept Maps; Software.

Resumen: El uso de la teoría de la enseñanza / aprendizaje en el desarrollo de objetos de aprendizaje de física básica en los cursos de ingeniería a menudo no es explícito. En este contexto, se justifica el desarrollo de objetos de aprendizaje, donde esta teoría es la base para su desarrollo con aportes significativos al Máster Profesional en Educación Científica y Tecnológica Este artículo tiene como objetivo informar sobre el desarrollo de un prototipo de software didáctico, para ser utilizado en el componente curricular de circuitos eléctricos. La metodología, en cuanto a su naturaleza, se puede clasificar como aplicada, la cual se caracteriza por su interés práctico, en que los resultados se aplican o se utilizan de manera inmediata en la solución de problemas que se han presentado en la realidad. En cuanto a los objetivos, se puede clasificar como exploratorio. En cuanto al abordaje del problema, se clasificó como una combinación de aspectos de la investigación cualitativa y cuantitativa, en cada etapa del proceso de investigación. Desde el punto de vista de los métodos, la investigación se realizó mediante modelado y simulación. Este estudio trae aportes para construir objetos de aprendizaje que puedan colaborar con el aprendizaje significativo de conceptos, tanto de física como de matemáticas, lo cual se enmarca en el propósito de una enseñanza científica y tecnológica.

Palabras-clave: Aprendizaje significativo; Circuitos electricos; Mapas conceptuales; Software.

\section{INTRODUÇÃO}

Enquanto o número de cursos de engenharia vem aumentando, a quantidade de professores das áreas de química, física e matemática (disciplinas básicas) está diminuindo. Assim, há uma redução na qualidade do ensino dos cursos de engenharia, em geral, haja vista os resultados obtidos pelos concluintes desses cursos no Exame Nacional de Desempenho de Estudantes (ENADE).

Nesse contexto, surge a justificativa de desenvolvimento de métodos e ferramentas que facilitem o aprendizado dos aprendizes. Sendo assim, este artigo tem como objetivo 
relatar o desenvolvimento de um protótipo de software de ensino a ser utilizado no componente curricular de circuitos elétricos, especialmente para análise de circuitos RC, RL e RLC. Apesar de os cursos de engenharias abrangerem conteúdos de várias áreas, que vão desde as ciências sociais até computação, passando pelas ciências exatas e da terra, este trabalho trata apenas do ensino de equações diferenciais e circuitos elétricos, que são temas relevantes, tanto no ponto de vista matemático quanto no ponto de vista físico da engenharia elétrica e sua aplicação a conteúdos práticos. Em estudo anterior usando aprendizagem significativa abordamos a interpretação para língua brasileira de sinais de terminologias matemáticas usadas na geometria plana e espacial como instrumento facilitador ao intérprete de sinais e ao professor de matemática, que trabalham com alunos surdos (SMOLSKI, et al., 2020).

Questionamo-nos como devemos desenvolver um objeto de aprendizagem, que teoria de ensino devemos utilizar como base? Esta é uma pergunta importante que devemos pensar antes de começarmos o desenvolvimento da pesquisa para produzir objetos de aprendizagem.

Para o desenvolvimento pleno da ciência da engenharia é importante compreender fenômenos físicos através de uma linguagem matemática adequada, para que seja possível utilizá-la na vida diária, ou seja, praticar uma engenharia moderna e de tecnologia avançada. Surge como uma das possíveis soluções para o ensino de componentes curriculares, no curso de engenharia elétrica, o estudo interdisciplinar, que é uma estratégia que vem sendo utilizada em universidades e instituições de pesquisa de países onde disciplinas que comutam conceitos teóricos são agrupadas em áreas antes não existentes. Assim, devemos demonstrar não somente que este tipo de estudo é importante, mas também desenvolver metodologias para que realmente possamos trabalhar a integração de conceitos (ZORLU, 2020). O ensino da disciplina de circuitos elétricos, nos cursos de graduação, dá-se através da apresentação de métodos de resolução de tipos de equações diferenciais integráveis, com a aplicação de listas de exercícios, os quais podem ser resolvidos pelos métodos apresentados, tornando-os, assim, um ensino que não apresenta significado. A autora completa, ainda, que a abordagem a qual privilegia processos algébricos determina a solução analítica, e, por muitas vezes, acaba minimizando a interpretação e o comportamento da solução do modelo analisado. Esta realidade, onde é trabalhado apenas o método analítico, acaba por caracterizar um processo mecânico de aprendizagem, e não significativo, pois o estudante aprende por execução e repetição de exercícios, não contextualizando o ensino dessas disciplinas, equações diferenciais e os conceitos físicos.

Recebido em: 30/05/2021

Aceite em: 13/08/2021 
Este estudo traz contribuições para atingir o objetivo de construir objetos de aprendizagem que possam colaborar com a aprendizagem significativa de conceitos, tanto de física como de matemática, associados às dinâmicas exigidas na parte profissionalizante de cursos de engenharia elétrica. Também como objetivos, mais específicos, desenvolver novas metodologias de ensino associadas à Física, Matemática e a Circuitos Elétricos. Igualmente, mostrar o desenvolvimento alternativo da demonstração das principais equações que regem o comportamento de circuitos elétricos, mais precisamente, os circuitos que tenham dispositivos associados com o circuito, resistor, capacitor e indutor, nas suas diversas configurações.

Para isso, desenvolve-se, neste trabalho, uma proposta de ensino utilizando a aprendizagem significativa de Ausubel (2002). Dos Santos et al. (2020), em que os novos conhecimentos que se adquirem são relacionados aos conhecimentos prévios que o aprendiz possui. Serão utilizados os conceitos prévios de cálculo e física básica na aquisição dos conhecimentos novos de eletricidade e equações diferenciais, que integrem também com o eletromagnetismo clássico, utilizando, como auxílio de visualização, softwares como uma ferramenta auxiliar de ensino, tendo o apoio do uso de mapas conceituais associados às técnicas de resoluções de equações diferenciais. No trabalho de Tomm et al. (2021) foi proposto, com base nesse método de ensino e mapas conceituais, um protótipo de software de ensino como um modelo, para facilitar o ensino-aprendizagem de física, assim como será proposto aqui.

\section{BREVE REVISÃO DE CONCEITOS TEÓRICOS}

\subsection{INTERDISCIPLINARIDADE: INTERAÇÃO ENTRE ÁREAS DO CONHECIMENTO}

Quando falamos em interdisciplinaridade, estamos de algum modo nos referindo a uma espécie de interação, ligação entre disciplinas ou áreas do saber (afins ou aparentemente não). Fazenda (2002) nos apresenta um breve histórico da interdisciplinaridade, mencionando seu surgimento na França e Itália, em meados de 1960, como um modelo de resposta a reivindicações por um ensino mais contextualizado, relacionado com as grandes questões de ordem social, política e econômica da época. Ao final da década de 60, a interdisciplinaridade chegou ao Brasil e logo exerceu influência na elaboração da Lei de Diretrizes e Bases $\mathrm{N}^{\circ}$ 
5.692/71 (SAVIANI, 2008). A partir de então, sua presença no cenário educacional brasileiro tem se intensificado ainda mais com a nova LDB N N $^{\circ}$ 9.394/96 e com os Parâmetros Curriculares Nacionais (PCNs).

Um fator de grande importância da interdisciplinaridade dá-se no momento em que ela oferece uma nova postura diante do conhecimento, uma nova maneira de construí-lo, de forma globalizada, integral, sem os tradicionais limites das disciplinas. Há grandes contribuições para o processo ensino-aprendizagem através da interdisciplinaridade: estudantes aprendem a trabalhar em grupo, habituam-se à experiência de aprendizagem integral. Professores, por sua vez, ampliam seus conhecimentos em outras áreas e minimizam os problemas de interação com os colegas de trabalho. Ainda, segundo Hamze (2009), outra característica do trabalho interdisciplinar condiz com o método envolvendo integração de conteúdos, o qual apresenta uma concepção unitária, e não mais fragmentada, do conhecimento, "ponderando sobre o estudo e a pesquisa, a partir do apoio das diversas ciências tornando o processo ensino-aprendizagem mais significativo" (HAMZE, 2009, p. 1).

O trabalho interdisciplinar é envolvente, mesmo que o professor tenha formação segmentada nos princípios que norteiam esta prática docente, citados por Moran, Masetto e Behrens (2000), a saber: humildade, espera, respeito, coerência e desapego - tornam o sentido de encontro da educação real e voltada à transdisciplinaridade. Ainda assim, destacam-se outras linhas escritas por esse autor: a confiança e a humildade do profissional docente que ensina e está atento para aprender o novo, é flexível e valoriza a diferença.

\subsection{MAPAS CONCEITUAIS APLICADOS À ENGENHARIA ELÉTRICA}

A construção de mapas conceituais, na maneira proposta por Novak e Gowin, considera uma estruturação hierárquica dos conceitos que devem ser apresentados, tanto de uma maneira que permitam uma diferenciação progressiva quanto de uma reconciliação integrativa (NOVAK, 1998; NOVAK; GOWIN, 1999).

A diferenciação progressiva é quando um determinado conceito é dividido em outros conceitos que estão contidos dentro do primeiro (em parte ou integralmente). Na reconciliação integrativa, um conceito de um ramo da raiz é relacionado a outro conceito de outro ramo da raiz, propiciando uma reconciliação, uma conexão entre conceitos que não era claramente perceptível por meio de palavras-chave.

Recebido em: 30/05/2021

Aceite em: 13/08/2021 
De um modo geral, para a construção de um mapa conceitual, o tema principal é registrado no topo por meio de um conceito e logo abaixo são organizados os demais conceitos que tem alguma relação com o assunto principal. Os conceitos sempre são escritos em algum tipo de figura, geralmente retângulos ou elipses e estes estarão unidos por meio de uma seta, que tem a função de estabelecer a relação entre os elementos conceituais (ANDREOLA; KRAUSE; FRANZIN, 2020, p.875).

Mapas conceituais podem seguir um modelo hierárquico, no qual conceitos mais inclusivos estão no topo da hierarquia (parte superior do mapa) e conceitos específicos, pouco abrangentes, estão na base (parte inferior). Mas esse é apenas um modelo, mapas conceituais não precisam necessariamente ter esse tipo de organização.

\subsection{APRENDIZAGEM SIGNIFICATIVA MODERNA}

Um dos principais autores modernos é Moreira (2006), que nos explica a existência de três formas de aprendizagem significativa: subordinada, superordenada e combinatória. Subordinada é quando um novo conhecimento é aprendido a partir de um conhecimento prévio já existente na estrutura cognitiva do aprendiz. A superordenada ocorre quando um conhecimento passa a ser hierarquicamente superior. Isso, normalmente, verifica-se quando o aprendiz faz ligações entre conhecimentos aprendidos. Por último, a combinatória, é quando ocorre uma interação cognitiva de um conjunto de conhecimentos prévios, formando novos conhecimentos (MOREIRA, 2013). Moreira nos diz que o que ocorre entre conhecimentos prévios e conhecimentos novos é uma interação cognitiva. O termo "ancoragem" é metafórico, porque nessa interação o "ancoradouro" também se modifica.

A aprendizagem significativa ocorre quando a tarefa da aprendizagem implica relacionar, de forma não arbitrária e substantiva, uma nova informação a outras com as quais o aluno já esteja familiarizado, e quando o aluno adota uma estratégia correspondente, para assim proceder (MEGGIOLARO; SANTOS, 2020, p. 51).

Resumidamente, o processo pode ser assim descrito: um novo conhecimento interage com algum conhecimento prévio, especificamente relevante, e o resultado disso é que esse novo conhecimento adquire significado para o aprendiz e o conhecimento prévio adquire novos significados, fica mais elaborado, mais claro, mais diferenciado, mais capaz de funcionar como subsunçor para outros novos conhecimentos. Durante certo período de tempo, a fase de retenção, o novo conhecimento pode ser reproduzido e utilizado com todas as suas características, independente do subsunçor que lhe deu significado em um processo de interação cognitiva. No entanto, simultaneamente, tem início um processo de obliteração, cujo resultado é um esquecimento (residual) daquele que era um novo conhecimento e que foi Recebido em: 30/05/2021

Aceite em: 13/08/2021 
aprendido significativamente. Isso quer dizer que aprendizagem significativa não é sinônima de "nunca esquecer" ou "daquilo que não esquecemos". A assimilação obliteradora é a continuidade natural da aprendizagem significativa, mas essa obliteração não leva a um esquecimento total. Ao contrário, o novo conhecimento acaba "ficando dentro do subsunçor" e a reaprendizagem é possível e relativamente fácil e rápida (MOREIRA, 2013).

Além das três formas de aprendizagem significativa, Moreira inclui também três tipos de aprendizagem significativa: representacional, conceitual e proposicional conforme Figura 1. Representacional é aquela em que um símbolo, um signo, um ícone, representa um único evento ou objeto. Conceitual quando o aprendiz consegue apontar regularidades em eventos ou objetos construindo assim um conceito. Proposicional ocorre quando o aprendiz consegue montar uma proposição que envolve os conceitos de determinado evento ou objeto.

\section{Figura 1 - Mapa Conceitual Ilustrando Formas e Tipos Aprendizagem Significativa.}

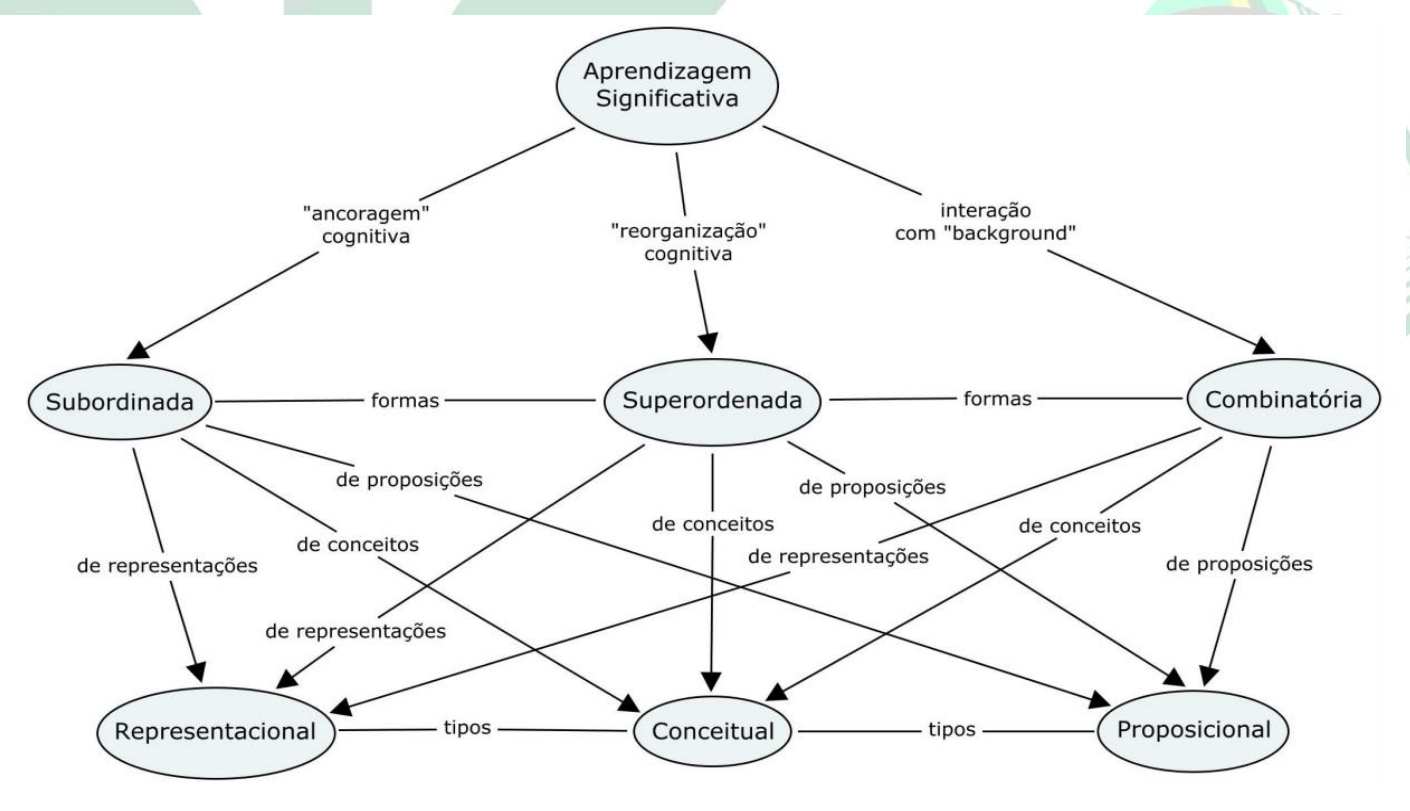

Fonte: (MOREIRA, 2013).

Sendo assim, as três formas de aprendizagem significativa: subordinada, superordenada e combinatória, compartilham os mesmos três tipos de interação cognitiva: a representacional, conceitual e proposicional.

\section{METODOLOGIA}

Recebido em: 30/05/2021

Aceite em: 13/08/2021 
Classificar a pesquisa é algo discutível, pois há vários autores. Esta pesquisa teve como base a pesquisa de João Batista Turrioni sobre metodologia (TURRIONI; MELLO, 2012). A seguinte classificação foi usada: Quanto à sua natureza, a pesquisa pode ser classificada como uma pesquisa aplicada, que se caracteriza pelo seu interesse prático, em que os resultados sejam aplicados ou utilizados imediatamente na solução de problemas ocorridos na realidade. Quanto aos objetivos, a pesquisa pode ser classificada como exploratória, visto que a pesquisa exploratória visa proporcionar maior familiaridade com o problema para explicitá-lo ou construir hipóteses. Quanto à abordagem do problema, o estudo foi classificado como uma combinação de aspectos da pesquisa qualitativa e quantitativa, em cada etapa do processo. Do ponto de vista dos métodos, a pesquisa foi feita por meio de modelagem e simulação. A modelagem e a simulação são utilizadas quando se tenta experimentar, por meio de um modelo complexo. Seu princípio está na construção de um modelo conceitual, no caso, o software de ensino.

Para a criação do software utilizado neste trabalho, a metodologia escolhida foi a Estruturada, que tem por objetivo retratar o fluxo e o conteúdo das informações utilizadas pelo sistema, ou seja, dividir o sistema em partições ambientais e comportamentais, descrevendo a essência do que será construído, uma vez que na Engenharia de Software as principais abordagens das Metodologias de Desenvolvimento de Software são a Metodologia Estruturada, a Metodologia Orientada a Objetos $\mathrm{e}$ as Metodologias Ágeis de Desenvolvimento.

O software foi aplicado com aprendizes do curso de Engenharia Mecânica, por meio da disciplina de Física Geral C, como material complementar nas aulas. Desta forma, o primeiro trabalho em aula foram os conceitos de carga, campo elétrico, potencial elétrico e lei de Gauss. E então foi fornecido o link do software para realização da atividade em casa.

O software foi construído seguindo os ensinamentos de Ausubel, nos quais a pretensão do educador é ensinar de forma significativa, onde devemos avaliar o que o aprendiz já sabe e depois ensinar, de acordo com esse conhecimento. A partir deste link http://srvapp2s.santoangelo.uri.br/ mect_survey/, os aprendizes da página inicial deveriam se cadastrar como usuário, aceitando ou rejeitando o termo de consentimento. Depois, o software apresenta ao aprendiz um teste inicial, cujo objetivo é avaliar os conceitos que os aprendizes 
já tiveram. Ao avançar na atividade, é apresentado ao aprendiz um Mapa de Conceitos, demonstrando ao aprendiz em quais conceitos o mesmo teve dificuldades.

De acordo com Booch, Rumbaugh e Jacobson (2000), um cenário é uma sequência específica de ações que ilustra o comportamento. Nesse caso, um cenário trata de uma breve descrição do que o sistema fará com seus personagens e ações principais. Pensando nisso, o aprendiz deve primeiro se cadastrar no sistema e autenticar seu cadastro. Em seguida, é gerado um formulário de consentimento, que deve ser aceito para que possam continuar sua experiência no software. Em seguida, os aprendizes respondem a uma pesquisa em que o sistema avalia as dificuldades dos aprendizes. O próximo cenário é um mapa conceitual mostrando as disciplinas nas quais os aprendizes têm mais dificuldade. Ao inserir um dos pontos dados, o sistema fornece aos aprendizes três maneiras diferentes de aprender o conteúdo, que são ler sobre o assunto, assistir a um vídeo ou fazer uma simulação do assunto. Ao final, os aprendizes são incentivados a refazer o levantamento inicial para que seu aprendizado seja analisado. Primeiros Cenários: Os aprendizes cadastram-se no sistema; Os aprendizes realizam a autenticação no sistema; O sistema pede o consentimento; Ao consentir, o aprendiz realiza a pesquisa inicial; O software apresenta um mapa conceitual com o conteúdo que os aprendizes precisam estudar; Os aprendizes estudam o conteúdo; Os aprendizes fazem a final.

\section{DESENVOLVIMENTO DO SOFTWARE}

Considerando as deficiências das ferramentas de ensino dos circuitos elétricos existentes e a necessidade de haver uma interdisciplinaridade entre os conceitos abordados, o software em desenvolvimento neste trabalho dispõe de uma orientação ao aprendiz que o utiliza. Para dispor de uma ferramenta completa, também se utilizou de ferramentas já existentes. Por exemplo, para as simulações de física, utilizou-se as simulações disponíveis no PhET. As calculadoras são as disponibilizadas pela Wolfram Alpha. Para o aprendizado por meio de vídeos, utilizou-se os disponibilizados pela plataforma do Youtube e para o método de conteúdos expositivos, as aulas expositivas disponíveis no SlideShare. Assim, o software agrega o que cada ferramenta tem de vantagem em uma única plataforma, que busca auxiliar e direcionar o aprendiz em seu aprendizado, fazendo, assim, o papel de um orientador. 
A metodologia consiste em desenvolver a arquitetura do software, que contém: Engenharia do software; Cenários de ensino; Casos de uso; Diagramas de classes; Diagramas de sequência; Diagramas de Estados; Interface de apresentação.

\subsection{ENGENHARIA DE SOFTWARE}

O software é um elemento de sistema lógico, e não físico. Portanto, o software tem características que são consideravelmente diferentes das do hardware: 1. O software é desenvolvido e projetado por engenharia, não manufaturado no sentido clássico; 2. O software não se desgasta; 3. A maioria dos softwares é feita para um fim específico em vez de ser adaptada a partir de outros softwares existentes.

A engenharia de software compreende um conjunto de etapas que envolvem métodos, ferramentas e os procedimentos. Essas etapas, muitas vezes, são citadas como paradigmas da engenharia de software. Um paradigma de engenharia de software é escolhido tendo como base a natureza do projeto e da aplicação, os métodos e as ferramentas a serem usados, os controles e os produtos que precisam ser entregues (SOMMERVILLE, 2003).

\subsubsection{Cenários}

Segundo Booch, Rumbaugh e Jacobson (2000), um cenário é uma sequência específica de ações que ilustra o comportamento. Nesse caso, um cenário trata da descrição resumida sobre o que o sistema fará com seus principais personagens e ações (BOOCH; RUMBAUGH; JACOBSON, 2000). Primeiramente, o aprendiz deve cadastrar-se no sistema e, então, fazer a autenticação nele. Após a autenticação, o sistema apresenta ao aprendiz o termo de consentimento que o aprendiz deve aceitar para poder continuar a sua experiência no software. Então, o aprendiz tem que responder a uma sondagem, a fim de levantarmos os subsunçores. O próximo cenário possui um mapa conceitual, o qual aponta ao aprendiz os conceitos nos quais ele possui dificuldades. Ao entrar em um dos pontos, o sistema apresenta três meios de aprendizagem que o aprendiz prefere estudar o conteúdo, são eles: lendo o conteúdo, vendo um vídeo do conteúdo e fazendo simulação do conteúdo. Ao finalizar todos os pontos, o aprendiz é incentivado a responder novamente o questionário. Quando o aprendiz 
acertar todas as questões do teste, o software irá apresentar o mapa de conceitos de nível superior que, agora, tendo todos os conceitos-âncora, o aprendiz está apto a aprender.

\subsubsection{Cenários Primários}

O Aprendiz cadastra-se no sistema; faz a autenticação no sistema; o sistema pede o consentimento do aprendiz; ao consentir, o aprendiz faz o teste inicial; se o aprendiz errar alguma questão, o software apresenta um mapa conceitual com os pontos para o aprendiz estudar; ao terminar de estudar os pontos, ele pode refazer o teste, e se o aprendiz gabaritar o teste, ou quando fizer o teste novamente e acertar todas as questões, o software irá apresentar um mapa conceitual com os conteúdos do nível superior para que ele aprenda os novos conceitos.

\subsubsection{Casos de Uso}

Os casos de uso descrevem o que o sistema faz do ponto de vista do usuário. Em outras palavras, ele descreve as principais funcionalidades do sistema e a interação dessas funcionalidades com os usuários do mesmo sistema. Os casos de uso não demonstram em detalhes técnicos o que o sistema faz.

Diagramas de casos de uso são compostos basicamente por quatro partes: cenário: sequência de eventos que ocorrem quando um ator interage com o sistema; ator: usuário do sistema; caso de uso: é uma tarefa ou uma funcionalidade realizada pelo ator; e comunicação: é o que liga um ator com um caso de uso.

\subsubsection{Diagrama de Classes}

Os diagramas de classes da Linguagem de Modelagem Unificada (UML) são super conjuntos dos diagramas de Entidade-Relacionamento (ER), uma ferramenta básica de modelagem para projetos lógicos de banco de dados. Além de ter foco nos dados como os diagramas ER, o diagrama de classe permite a modelagem dos comportamentos dos dados (BOOCH; RUMBAUGH; JACOBSON, 2000). 
O diagrama de classes descreve um conjunto de objetos que compartilham os mesmos atributos, operações e relacionamentos. As classes são os blocos fundamentais de qualquer sistema orientado a objeto.

As classes possuem três atributos principais: nome-identificador da classe; conjunto de atributos; e conjunto de métodos.

Entre as classes, existem relacionamentos. Os três principais relacionamentos são: a generalização, a agregação e a associação. O diagrama do software apenas possui a terceira, associação, que, somente, identifica que há uma relação entre as classes. A cardinalidade, número entre os relacionamentos que demonstra quantas ocorrências uma classe pode ter com a outra. A Figura 2 demonstra o diagrama de classes do software.

Figura 2 - Diagrama de Classes do Software.

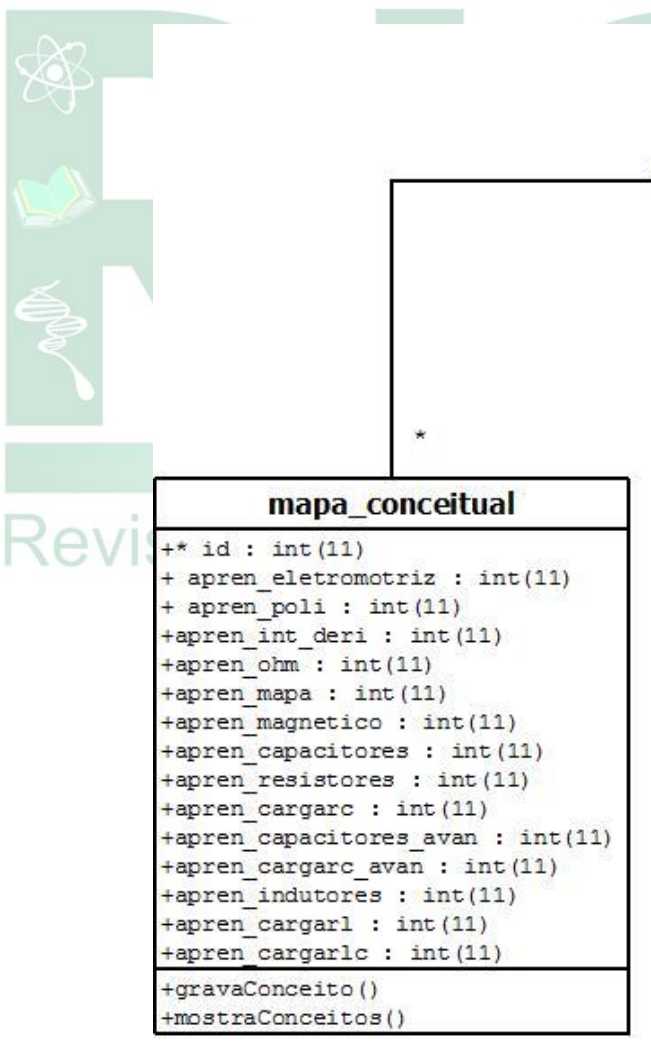

\begin{tabular}{|c|}
\hline usuarios \\
\hline $\begin{array}{l}\text { +* id : int }(10) \\
\text { +usuario: varchar }(50) \\
\text { +senha: varchar }(50) \\
\text { +status : int (11) } \\
\text { +termo: int (11) }\end{array}$ \\
\hline $\begin{array}{l}\text { +gravaUsuario() } \\
\text { +buscaUsuario() } \\
\text { +atualizaUsuario() }\end{array}$ \\
\hline $\begin{array}{l}1 \\
*\end{array}$ \\
\hline questionario_final \\
\hline $\begin{array}{l}\text { +QuestaoID : int (11) } \\
\text { +Questao1: tinyint (4) } \\
\text { +Questao2 : tinyint (4) } \\
\text { +Questao3: tinyint (4) } \\
\text { +Questao4: tinyint (4) } \\
\text { +Questao5: tinyint (4) } \\
\text { +Questao6 : tinyint (4) } \\
\text { +Questao7: tinyint (4) } \\
\text { +Questao8: tinyint (4) } \\
\text { +Questao9: tinyint (4) } \\
\text { +Questao10: tinyint (4) } \\
\text { +Questao11: tinyint (4) } \\
\text { +Questao12: tinyint (4) } \\
\text { +Questao13: tinyint (4) } \\
\text { +Questao14: tinyint (4) }\end{array}$ \\
\hline $\begin{array}{l}\text { +gravaQuestoes () } \\
\text { +buscaQuestoes () }\end{array}$ \\
\hline
\end{tabular}

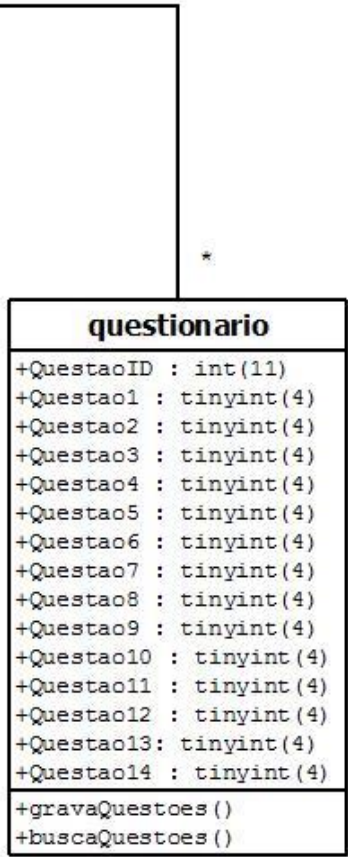

Fonte: Autores (2021).

\subsubsection{Diagrama de Sequência}

Recebido em: 30/05/2021

Aceite em: 13/08/2021 
Um diagrama de sequência descreve a maneira como os grupos de objetos colaboram em algum comportamento, ao longo do tempo. Ele registra o comportamento de um único caso de uso e exibe os objetos e as mensagens passadas entre esses objetos nos casos de uso.

A notação para uma mensagem em um diagrama de sequência é uma flecha, ligando uma linha de vida à outra. O objeto do qual parte a seta é aquele que está enviando a mensagem. $\mathrm{O}$ objeto para o qual a seta aponta é aquele que está recebendo a mensagem. $\mathrm{O}$ formato da ponta da seta indica o tipo de mensagem sendo enviada. O rótulo da mensagem é posicionado acima das setas.

As linhas de vida - linhas verticais - representam o tempo de vida de um objeto. Uma linha de vida é composta de duas partes, a cabeça e a cauda.

A cabeça é representada por um retângulo com dois compartimentos: no compartimento superior, a identificação do objeto é exibida; e a cauda corresponde a uma linha vertical tracejada. A Figura 3 ilustra o diagrama de sequência do software.

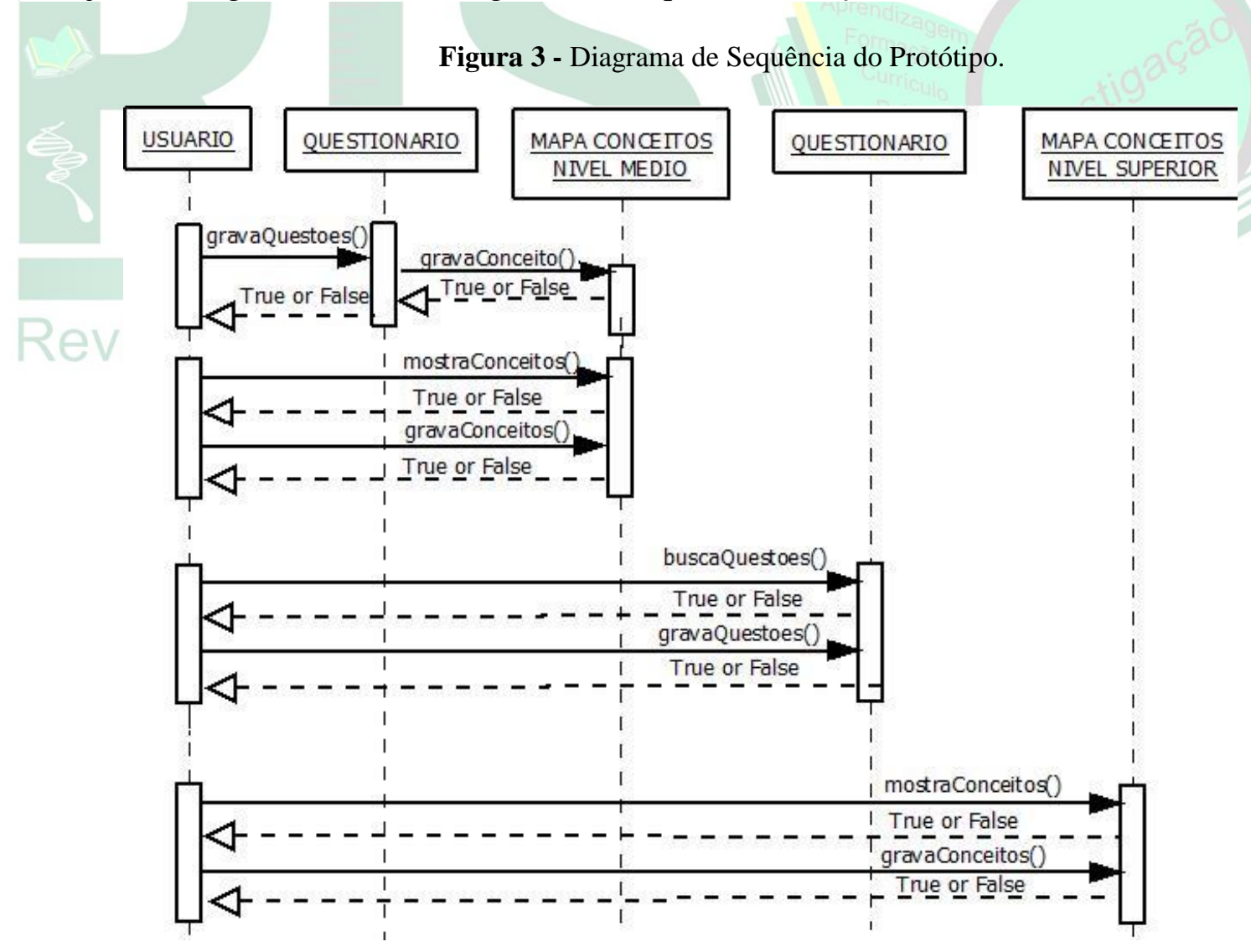

Fonte: Autores (2021).

Recebido em: 30/05/2021

Aceite em: 13/08/2021 


\subsubsection{Diagrama de Estados}

Uma das atividades mais importantes da etapa de desenvolvimento é a validação dos estados pelos quais um objeto deve passar. Compreender a ordem temporal e a sequência com que as transições de estados acontecem, permite uma validação mais eficiente e um domínio da solução mais abrangente.

Em um diagrama de estado, um objeto possui um comportamento e um estado. O estado de um objeto depende da atividade na qual ele está processando. Um diagrama de estado mostra os possíveis estados de um objeto e as transações responsáveis pelas suas mudanças de estado. A Figura 4, a seguir, demonstra o diagrama de estados do sistema.

Figura 4 - Diagrama de Sequência do Protótipo.

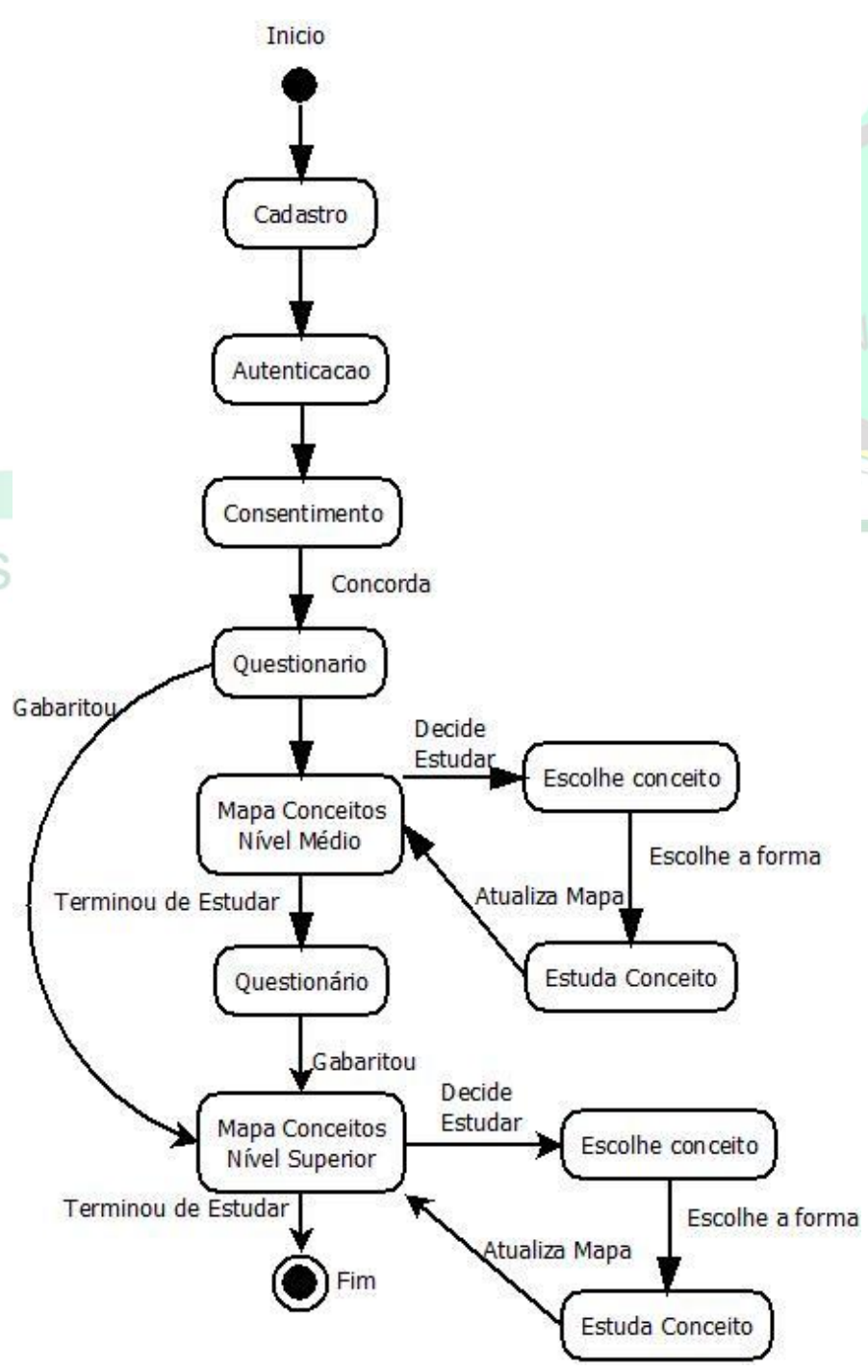

Fonte: Autores (2021).

Recebido em: 30/05/2021

Aceite em: 13/08/2021 
O teste inicial aborda os conceitos básicos de circuitos RLC que os estudantes aprenderam no ensino médio. Aborda, também, questões para testar os conhecimentos matemáticos básicos, necessários para solucionar os problemas da disciplina em questão.

Depois de aplicado o teste, o software para circuitos analisa as respostas dos aprendizes para buscar os pontos em que os aprendizes apresentam carência, para, assim, poder suprir essas deficiências. Após a análise, o software expõe um mapa conceitual desses conceitos de nível médio, abordados no teste, onde realça os pontos em que o aprendiz precisa reforçar seus conhecimentos. Ao acessar, clicando em um dos pontos dentro do mapa conceitual, o protótipo indica três opções de como o aprendiz pode estudar o conteúdo, são elas: lendo, vendo ou fazendo simulação sobre o assunto. Depois de feita a escolha, o material de apoio é apresentado para que os aprendizes consigam obter esses pré-conceitos, nos quais residem suas dificuldades. A eliminação dessas deficiências é necessária para se atingir a aprendizagem significativa dos conceitos.

O aprendiz, após estudar, deve refazer o teste inicial com a finalidade de evidenciar se ele ainda possui dificuldades nesses conteúdos. Se o aprendiz acertar todas as questões, então ele será redirecionado para o mapa de conceitos de nível superior, o mesmo ocorre se o aprendiz gabaritar o teste inicial na primeira vez que o fizer. No mapa de nível superior, o aprendiz também deve estudar os conceitos, conforme anteriormente no mapa de conceitos do nível médio, escolhendo uma das três opções, segundo fora antes explanado. Os testes têm caráter quantitativo, com perguntas estruturadas, claras e objetivas, de múltipla escolha. As linguagens de programação utilizadas para a programação do protótipo foram $P H P, H T M L$,

\section{LOG IN E CADASTRO ENSINO DE CIRCUITOS RLC}

Clique "Cadastrar-se" para ir para página de cadastro.

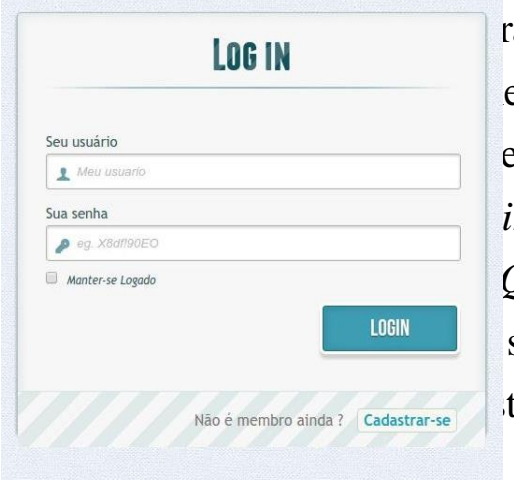

Fonte: Autores (2021). ra rodar a aplicação foi o Apache, por ser o melhor servidor web ento de banco de dados (SGBD) utilizado foi o $M y S Q L$, por ser erto mais popular para a $W e b$, rápido, confiável e fácil de usar in, que é um aplicativo gratuito da web desenvolvido em PHP $Q L$. O software possui também um sistema de cadastro e senha para manter a segurança e privacidade dos dados de cada :tema de cadastro e autenticação está demonstrado na 
Figura 5 - Página Inicial de Autenticação do Usuário

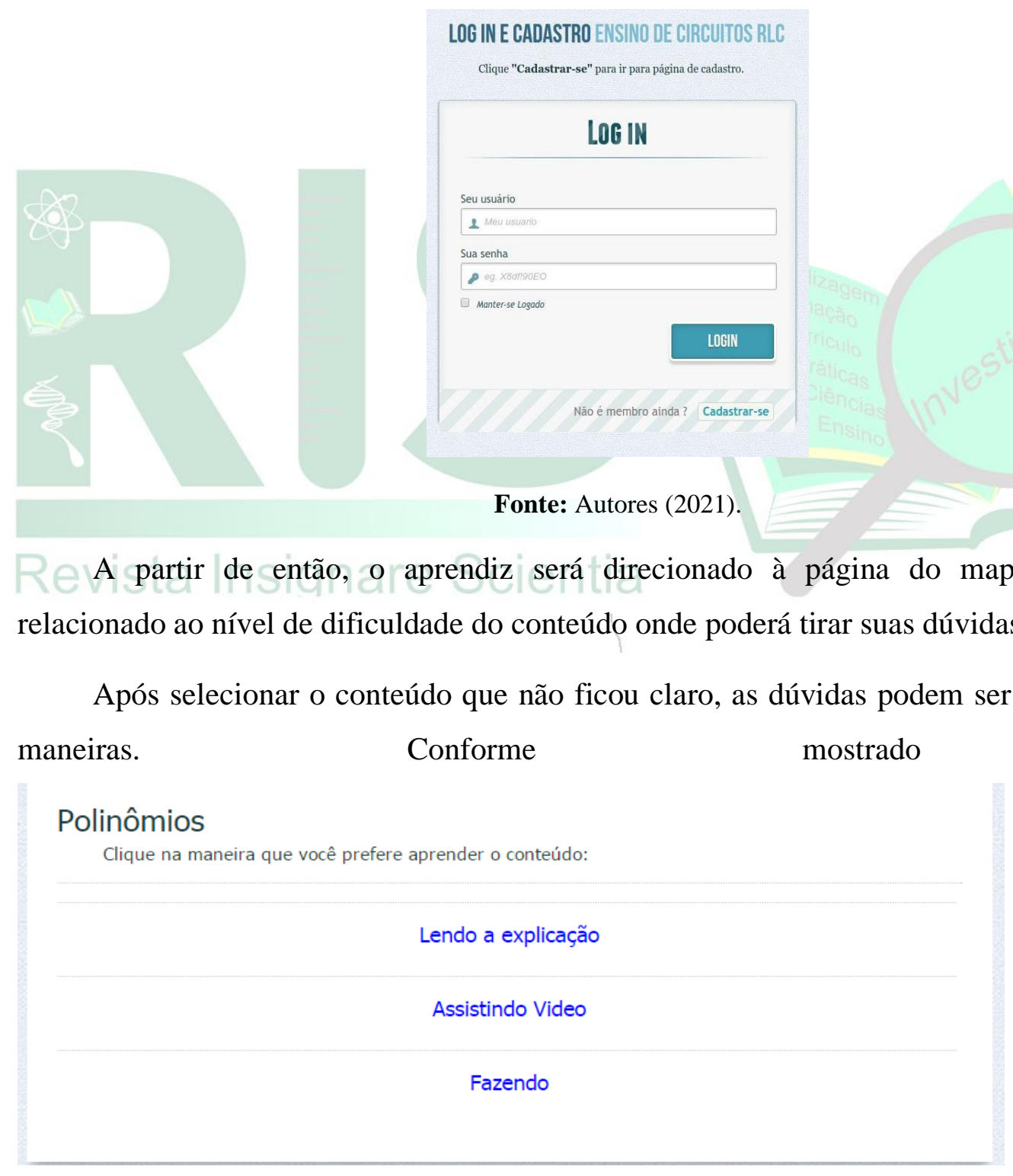

Recebido em: 30/05/2021

Aceite em: 13/08/2021 
6, o aprendiz tem a opção de escolher entre aprender: lendo a explicação, assistindo vídeos sobre o conteúdo, ou, então, fazendo simulações.

Figura 6 - Exemplo de Página de Escolha da Forma de Aprendizado.

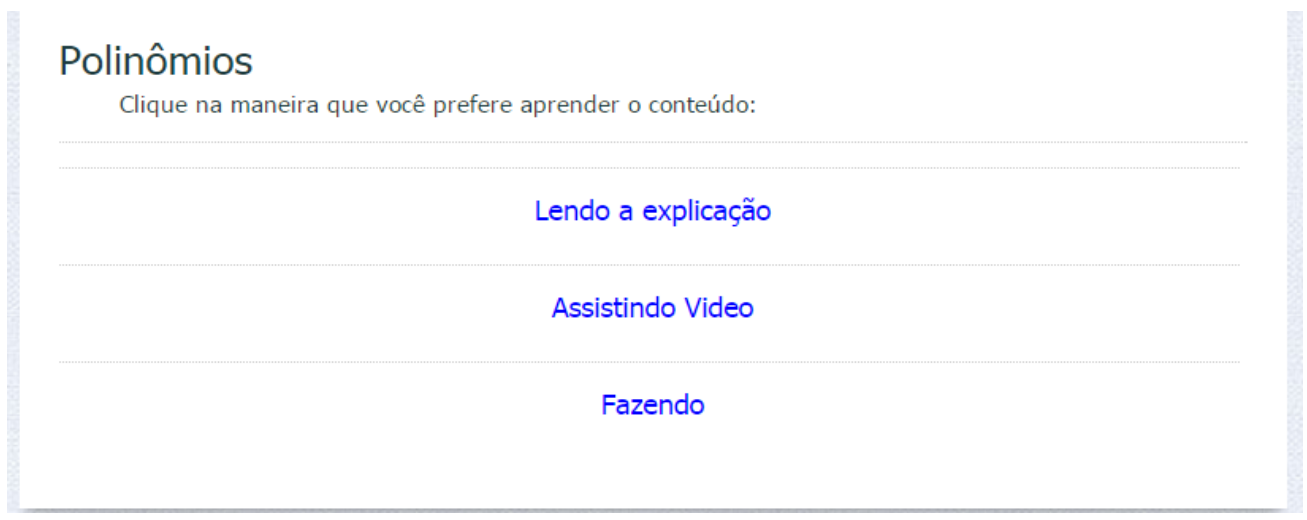

Fonte: Autores (2021).

A ferramenta desenvolvida é constituída de diversas páginas WEB. Por meio de um navegador (Google Chrome, Mozilla Firefox ou Opera), o aprendiz consegue acessar e interagir com o software, que orienta qual o próximo assunto que deve ser estudado, disponibilizando os conteúdos da forma que o aprendiz preferir aprender: lendo a explicação, assistindo vídeo ou fazendo uma simulação.

A utilização do protótipo de software desenvolvido poderá facilitar o ensino e a aprendizagem de circuitos elétricos, tendo por base o método de ensino da aprendizagem significativa de Ausubel e os mapas conceituais de Novak. Este artigo demonstra claramente o relevante papel da pesquisa na formação dos profissionais da educação, com significativas contribuições para o Mestrado Profissional de Ensino Científico e Tecnológico.

\section{CONSIDERAÇÕES FINAIS}

O objetivo de desenvolver um protótipo de software para a facilitação do ensino e da aprendizagem de circuitos elétricos, tendo por base o método de ensino da aprendizagem significativa de Ausubel e os mapas conceituais de Novak, foi alcançado. O protótipo poderá desempenhar papel relevante na formação dos profissionais da educação, com significativas contribuições para o Mestrado Profissional de Ensino Científico e Tecnológico. 
Sabendo que o ensino de circuitos elétricos é de fundamental importância na formação acadêmica dos engenheiros eletricistas, e que esse componente curricular serve de base aos outros de formação profissional, é essencial que o aprendiz seja aprovado na disciplina com o mínimo de dúvidas possíveis. Desta forma, o software proposto neste trabalho deve contribuir para o desenvolvimento dos aprendizes dos cursos de engenharia elétrica. Para os docentes, o protótipo desenvolvido serve como ferramenta facilitadora para o entendimento e aplicação dos conceitos repassados em sala de aula.

Utilizar ferramentas de simulação e conteúdos oferecidos por outras plataformas de ensino tornou o software mais completo e de fácil adaptação aos aprendizes, que normalmente já tem familiaridade com os layouts das plataformas mencionadas. Por outro lado, através da organização dos conteúdos, o software desenvolvido proporciona orientação aos aprendizes, o que se torna um diferencial.

À medida que o software vai sendo utilizado pelos aprendizes, é possível fazer uma análise comparativa do rendimento dos aprendizes, com e sem o auxílio da ferramenta. As conclusões sobre a eficiência da ferramenta também podem ser obtidas através dos depoimentos dos aprendizes.

O emprego de novas tecnologias de ensino nos cursos de engenharia deve ser aplicado não só nas disciplinas de formação básica, mas também nas disciplinas de formação profissionalizante. Desta forma, pode-se aplicar a metodologia proposta neste trabalho para desenvolver softwares de facilitação aos aprendizados das demais disciplinas do curso de engenharia elétrica e das demais engenharias. Além disso, é interessante fazer um levantamento e análise estatística do desempenho do software desenvolvido neste trabalho.

\section{REFERÊNCIAS}

ANDREOLA, C. S; KRAUSE, J. C; FRANZIN, R. F. Usando Mapas Conceituais para uma Aprendizagem Significativa de Geometria. Revista Brasileira de Ensino de Ciências e Matemática - RBECM. Passo Fundo, v. 3, n. 3, p. 868-889, ed. espec. 2020. Disponível em: http://seer.upf.br/index.php/rbecm/article/view/10761/114115554. Acesso em: 12 dez 2020.

AUSUBEL, D. P. Adquisición y retención del conocimiento. Una perspectiva cognitiva. Barcelona: Paidós, 2002.

BOOCH, G; RUMBAUGH, J; JACOBSON, I. UML - Guia do Usuário. S. 1. Campus, 2000.

Recebido em: 30/05/2021

Aceite em: 13/08/2021 
DOS SANTOS, A.V.; KILLIAN, P.; DA ROSA, C. T. W. Análise bibliométrica da produção científica nas bases de dados Scopus e Web of Science sobre Aprendizagem Significativa. Revista Insignare Scientia, v. 3, p. 443-459, 2020.

FAZENDA, I. C. A. (Org.). Dicionário em construção: interdisciplinaridade. 2 ed. São Paulo: Cortez, 2002.

HAMZE, A. Repaginando a Educação Infantil. (2009). Disponível em: http://www.educador.brasilescola.com/gestao-educacional/repaginando-aeducacaoinfantil.htm. Acesso em: 12 dez 2020.

G.I.R.E.P. Conference on Physics Education, Utrecht, p. 189-214, 1984.

MEGGIOLARO, G. P; SANTOS, A. V. Uma proposta de mapas conceituais a partir da abordagem do conceito de campo elétrico em livros didáticos. VIVÊECIAS (URI.

ERECHIM), v. 6, p. 47-47, 2020. Disponível em:

http://revistas.uri.br/index.php/vivencias/article/view/103. Acesso em: 12 dez 2020.

MORAN, J. M; MASETTO, M. T; BEHRENS, M. A. Novas tecnologias e mediação pedagógica. Campinas, SP: Papirus, 2000.

MOREIRA, M. A. A teoria da aprendizagem significativa e sua implicação em sala de aula. Brasília: Ed. UnB, 2006.

MOREIRA, M. A. Aprendizagem significativa em mapas conceituais. Porto Alegre: UFRGS, Instituto de Física, 2013.

NOVAK, J. D. Conocimiento e Aprendizaje: Los mapas conceptuales como herramientas facilitadoras para escuelas y empresas. Madrid: Editorial Alianza, 1998.

NOVAK, J. D; GOWIN, D. B. Aprender a aprender. Lisboa: Plátano Edições Técnicas, 1999.

SAVIANI, D. A pedagogia no Brasil: história e teoria. Campinas, SP: Autores Associados, 2008.

SMOLSKI, L. C. J.; FRANZIN, R. F.; SANTOS, A. V.; STRACKE, M. P. Terminologias matemáticas em Libras: a geometria plana e espacial. Revista Insignare Scientia, v. 3, p. 249-269, 2020.

SOMMERVILLE, I. Engenharia de Software. 6 ed. São Paulo: Addison Wesley. 2003.

TOMM, C. F; SANTOS, A. V.; FRANZIN, R. F; STRACKE, M. P; MEGGIOLARO, G. P. Análise dos resultados de uma aplicação de um software de Ensino de Física. Brazilian Journal of Development. Curitiba, v.7, n.1, p.5862-5870 jan. 2021. Disponível em: https://www.brazilianjournals.com/index.php/BRJD/article/view/23242. Acesso em: $12 \mathrm{dez}$ 2020.

Recebido em: 30/05/2021

Aceite em: 13/08/2021 
TURRIONI, João Batista. MELLO, Carlos Henrique Pereira. Metodologia de Pesquisa em Engenharia de Produção: estratégias, métodos e técnicas para condução de pesquisas quantitativas e qualitativas. Universidade Federal de Itajubá (UNIFEI), 2012. Disponível em $<$ http://www.carlosmello.unifei.edu.br/Disciplinas/Mestrado/PCM-

10/ApostilaMestrado/Apostila_Metodologia_Completa_2012.pdf>. Acesso em: $12 \mathrm{dez} 2020$.

ZORLU, F; ZORLU, Y. Investigation of the Effects of a Peer-Led Team Learning Instructional Model (PLTL) in Teaching the Simple Electrical Circuits Subject on the Seven Principles for Good Practice. Educational Policy Analysis and Strategic Research. 2020, Vol. 15(3) 249-266.

Recebido em: 30/05/2021

Aceite em: 13/08/2021 